\title{
Práticas alimentares no primeiro ano de vida de filhos de adolescentes
}

\author{
Adolescents' infant feeding practices \\ in the first year of life
}

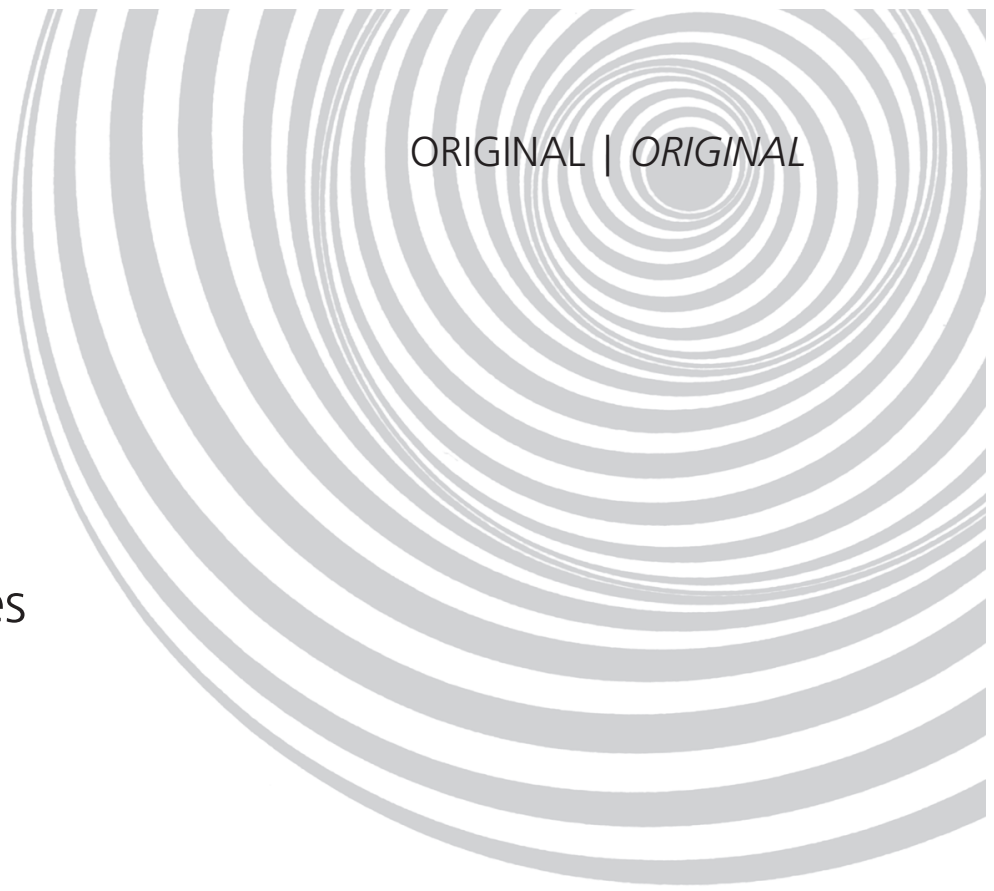

Myrian Coelho Cunha da CRUZ1

João Aprígio Guerra de ALMEIDA²

Elyne Montenegro ENGSTROM ${ }^{3}$

RE S U M O

\section{Objetivo}

Estudar as práticas alimentares de menores de um ano, filhos de adolescentes, residentes no município de Volta Redonda (RJ).

\section{Métodos}

Estudo transversal com 1014 crianças selecionadas aleatoriamente na Campanha de Multivacinação de 2006, com aplicação de questionário fechado (recordatório de 24h). Foram analisadas características das mães e de seus filhos. As prevalências de aleitamento materno, aleitamento materno exclusivo, aleitamento materno predominante, a oferta de outros leites, a alimentação no primeiro dia em casa, a alimentação complementar oportuna, a oferta de sólidos ou semi-sólidos, e o uso de chupetas e mamadeiras foram investigadas.

\section{Resultados}

As prevalências aleitamento materno, aleitamento materno exclusivo e aleitamento materno predominante foram $85,5 \%, 32,0 \%$ e 9,6\%, respectivamente, sem diferenças entre filhos de adolescentes e adultas. A oferta de outros leites, entretanto, foi significativamente maior junto aos filhos de adultas (45,3\%, contra 31,2\% observados entre adolescentes). Maiores de seis meses, filhos de adolescentes, revelaram menor prevalência de aleitamento materno que os de adultas (49,2\% e 66,0\%, respectivamente), assim como maior frequência no uso de chupetas, com diferenças significativas. A alimentação complementar foi similar entre filhos de adolescentes e adultas, com predominância da oferta de sopas ou papas. Carne e feijão foram utilizados com mais frequência em sólidos.

\section{Conclusão}

Não foram observadas diferenças no aleitamento considerando a variável idade materna, adolescente ou não, antes de seis meses de vida e sim após esse período. Nos maiores de seis meses, chama também a atenção o

\footnotetext{
1 Secretaria Municipal de Saúde de Volta Redonda. R. 566, n. 31, Nossa Senhora das Graças, 27295-390, Volta Redonda, RJ, Brasil. Correspondência para/Correspondence to: M.C.C. CRUZ.E-mail: <myriancruz@hotmail.com>.

2 Fundação Oswaldo Cruz, Instituto Fernandes Figueira. Rio de Janeiro, RJ, Brasil.

3 Fundação Oswaldo Cruz, Escola Nacional de Saúde Pública, Centro de Saúde Escola Germano Sinval Faria. Rio de Janeiro, RJ, Brasil.
} 
202 | M.C.C. CRUZ et al.

maior no uso de chupetas, assim como a oferta e as características da alimentação complementar de filhos de adolescentes. Estudos de natureza compreensiva mostram-se necessários.

Termos de indexação: Aleitamento materno. Alimentação complementar. Chupeta. Mamadeira. Mães adolescentes.

\section{A B S T R A C T}

\section{Objective}

The objective of this work was to study the feeding practices of adolescent mothers' infants under one year of age living in the city of Volta Redonda (RJ).

\section{Methods}

This cross-sectional study included 1,014 infants selected randomly at the 2006 Multiple Vaccination Campaign. The 24-hour recall was used. Characteristics of the mothers and infants were analyzed. The prevalences of breastfeeding, exclusive and predominant breastfeeding and the use of other kinds of milk, in addition to the feeding practices in the first day at home and use of complementary foods, solid or semi-solid foods and pacifiers and bottles were investigated.

\section{Results}

The prevalences of breastfeeding and exclusive and predominant breastfeeding were $85.5 \%, 32.0 \%$ and $9.6 \%$, respectively, with no differences between adolescents' and adults' children. Adult mothers were significantly more likely to offer other kinds of milk than adolescent mothers (45.3\% and 31.2\% respectively). Infants at six or more months of age of adolescent mothers were significantly less likely to be breastfed than those of adult mothers (49.2\% and 66.0\% respectively) and more likely to be given pacifiers. Adult and adolescent mothers presented similar complementary feeding practices, with soups and soft foods prevailing. Meat and beans were the most common solid foods given to the infants.

\section{Conclusion}

There are no differences in breastfeeding practices among mothers of different ages, whether adolescent or not, in the first six months of an infant's life but the practices do differ after this age. Among the six-monthold infants or older of adolescent mothers, the extensive use of pacifiers and the characteristics of the complementary foods deserve attention. Comprehensive studies are necessary.

Indexing terms: Breastfeeding. Complementary feeding.Pacifier. Bottle-feeding. Adolescent mothers.

\section{N TRO D U ÇÃ O}

Os avanços do conhecimento científico comprovam, cada vez mais, a importância da amamentação na ecologia do desenvolvimento humano. O leite humano é uma substância biologicamente ativa, capaz de diminuir a incidência e/ou gravidade dos eventos mórbidos da primeira infância, de proteger contra o óbito infantil' além de reduzir os riscos de ocorrência de doenças crônicas não transmissíveis na vida adulta². Seus nutrientes satisfazem às necessidades nutricionais do lactente, até o sexto mês de vida ${ }^{3}$. Tem a capacidade de preparar a criança para aceitar os novos alimentos, facilitando o estabelecimento de hábitos alimentares mais saudáveis em relação aos demonstrados por crianças não amamentadas ${ }^{4}$.
As práticas alimentares são construídas a partir de dimensões temporais, de saúde e de doença, de cuidado, afetivas, econômicas e de ritual de socialização, entrelaçadas em rede ${ }^{5}$. Os primeiros dois anos de vida da criança são caracterizados por crescimento acelerado e grande desenvolvimento psicomotor e neurológico. Logo, as deficiências nutricionais na primeira infância podem comprometer o padrão de crescimento, gerar atraso escolar e favorecer, futuramente, o surgimento de doenças crônicas ${ }^{6}$.

As recomendações sobre alimentação infantil resultam de um processo histórico, cujo eixo é a amamentação ${ }^{7}$. Os alimentos introduzidos na dieta infantil são reconhecidos, atualmente, como complementares ao leite humano e não mais como alimentos de desmame. A consistência 
da alimentação complementar, relacionada à satisfação das demandas nutricionais específicas dessa fase da infância, tem sido destacada. Para o sétimo e o oitavo mês de vida, recomenda-se a oferta de purê ou papa, ao passo que, aos oito meses, as refeições deverão apresentar maior consistência ${ }^{8}$. Preparações peneiradas ou liquidificadas são consideradas inadequadas ${ }^{9,10}$. Essas orientações, assumidas pelo Ministério da Saúde, definem, como prática alimentar ideal para lactentes, o aleitamento materno exclusivo até o sexto mês e complementado, a partir de então, até os dois anos de vida ou mais 9 .

A cada década, observa-se, no Brasil, uma sensível redução na proporção de crianças com déficits antropométricos ${ }^{11}$. No entanto maiores chances de déficits estaturais entre crianças brasileiras, filhas de adolescentes, foram evidenciadas $^{12}$. Apesar dos comprovados benefícios do aleitamento materno e dos esforços desenvolvidos no Brasil para sua prevalência, ela permanece baixa. A reanálise do estudo sobre a prevalência de aleitamento materno nas capitais brasileiras, realizado em 1999, revelou queda acentuada na prevalência de aleitamento materno exclusivo ${ }^{13}$. Embora a Pesquisa Nacional de Demografia e Saúde (2006) tenha evidenciado discreto aumento no aleitamento materno exclusivo entre menores de três meses, observou-se redução em sua prática entre crianças de quatro a seis meses ${ }^{14}$.

Nesse sentido, o presente estudo teve por objetivo analisar as práticas alimentares de lactentes, no primeiro ano de vida, residentes no município de Volta Redonda, Rio de Janeiro, assim como evidenciar as diferenças entre as aplicadas por mães adolescentes e adultas.

\section{MÉ T O D O S}

A pesquisa, do tipo transversal, voltou-se para a análise da prática alimentar dos lactentes menores de um ano, residentes no município de Volta Redonda. Para tal, foram utilizados os dados coletados em dias de Campanha Nacional de Multivacinação de 2006, de acordo com os pre- ceitos metodológicos do "Projeto Amamentação e Municípios"(AMAMUNIC), do Instituto de Saúde de São Paulo ${ }^{15}$. A amostra probabilística por conglomerados (postos de vacinação) foi calculada com base no número de menores de um ano vacinados no ano anterior. Foram consideradas 1014 entrevistas, contendo dados sobre idade materna, aleitamento e data completa do nascimento da criança, que correspondem a 35\% dos 2900 vacinados. O questionário, fechado, foi aplicado por universitários treinados e supervisionados por profissionais da Secretaria Municipal de Saúde. Os temas abordados nas entrevistas foram: alimentação das crianças nas 24 horas que antecederam a pesquisa; características do parto e nascimento; alimentação no primeiro dia em casa e características maternas.

Os aspectos biológicos e sociais das crianças e mães estudadas foram analisados por meio de variáveis, estruturadas em categorias. As idades das crianças compuseram dois grandes grupos: menor e maior ou igual a seis meses. Crianças nascidas com peso inferior a $2500 \mathrm{~g}$ foram consideradas com baixo peso, enquanto o tipo de parto foi classificado em normal (parto vaginal) e cesáreo ou fórceps. A paridade foi descrita em duas categorias: primeiro filho e segundo ou mais. Para a escolaridade materna foram criadas quatro categorias, construídas a partir da informação do último ano de estudo concluído: fundamental incompleto (até o sétimo ano); fundamental completo (que concluíram o oitavo ano); médio incompleto (até o segundo ano) e médio mais superior (concluíram o terceiro ano do ensino médio ou cursaram qualquer período do ensino superior). Foi investigado se a mãe da criança estudava ou não no momento da pesquisa e, em relação ao trabalho materno, se era doméstico (para as donas de casa), formal ou informal. A idade materna foi categorizada com base na cronologia, construindo dois grupos de mulheres/mães: até 19 anos completos (adolescentes) e com idades de 20 anos ou mais (adultas ou não adolescentes).

Os indicadores de aleitamento materno adotados foram os preconizados pela Organização 
Mundial da Saúde (OMS) ${ }^{16}$, referentes a práticas alimentares: de crianças menores de seis meses: Aleitamento Materno Exclusivo (AME): nenhum outro alimento é oferecido à criança, além do leite materno; Aleitamento Materno Predominante (AMP): além do leite materno, a criança recebe água, chás ou sucos; Aleitamento Materno (AM): considera as crianças que receberam leite materno, acompanhado ou não de outros leites e/ou alimentos. De crianças amamentadas, com idades entre seis e nove meses: Alimentação Complementar Oportuna (ACO): oferta de alimentos sólidos ou semi-sólidos.

Outros indicadores: oferta de água (Água) e oferta de chás (Chá): verificada no primeiro dia em casa, após a alta hospitalar; oferta de outros leites (Outros leites): observada no primeiro dia em casa e entre crianças menores de 6 meses, amamentadas ou não; Alimentação Complementar (AC): oferta de alimentos sólidos ou semi-sólidos, a crianças com idades de seis a doze meses.

Foram consideradas somente as entrevistas cujos dados relativos à idade materna e ao tipo de aleitamento materno são conhecidos ( $n=1014)$. Na presente amostra, 144 (14,2\%) entrevistas são provenientes de filhos de adolescentes e 870 $(85,8 \%)$ de adultas. Destas, 553 (54,5\%) referem-se aos menores de 6 meses e 461 (45,5\%) aos maiores de 6 meses. A descrição das práticas alimentares, no primeiro dia em casa, baseou-se nos dados levantados a partir de perguntas específicas aplicadas somente aos responsáveis por crianças menores de 4 meses $(n=383)$.

O programa AMAMUNIC foi aplicado para a definição de alguns indicadores, enquanto o programa Statistical Package for the Social Sciences (SPSS)-13.0 foi utilizado para as demais análises. O teste qui-quadrado, visando à comparação de proporções, foi aplicado na análise, com nível de significância estatística de 5\%. Esta pesquisa foi aprovada pelo Comitê de Ética do Centro Universitário de Volta Redonda (processo $\mathrm{n}^{\circ}$ 009/2008).

\section{RESULTADOS}

As características biológicas e sociais das crianças pesquisadas e de suas mães são apresentadas na Tabela 1 e revelam não existirem diferenças significativas em relação à distribuição etária das crianças e ao peso ao nascer entre filhos de adolescentes e não adolescentes. No entanto, diferenças significativas foram encontradas em relação à prevalência de parto normal, maior entre adolescentes, embora a proporção de cesarianas nesse grupo seja considerada elevada $(43,8 \%)$. Verificou-se que $17,6 \%$ das adolescentes tinham mais de um filho. Quanto à escolaridade materna, foi identificado que, no conjunto das entrevistadas, 25 mulheres/mães não sabiam ler e escrever. 0 ensino fundamental incompleto predominou entre as mulheres/mães adolescentes, enquanto a formação média e superior foi mais frequente entre as mulheres/mães adultas. Observou-se que $75,0 \%$ das adolescentes não eram estudantes no momento da pesquisa. O trabalho doméstico predominou entre todas as mães estudadas, especialmente no conjunto de adolescentes, com diferenças significativas entre os grupos.

A análise das práticas alimentares revela que as prevalências de aleitamento materno exclusivo aos 30 e 120 dias foram, respectivamente, 49,0\% e 27,4\% (resultados não apresentados em tabela). No primeiro ano de vida, a prevalência de aleitamento materno foi de $75,6 \%$, não sendo significativas as diferenças entre adolescentes e adultas (Tabela 1).

Entre os menores de seis meses, as prevalência de $A M, A M E, A M P$, do consumo de outros leites, assim como o uso de mamadeira e chupeta, está apresentada na Tabela 2. Entre filhos de adolescentes, as prevalências de AM, AME e AMP foram, respectivamente, de $90,4 \%, 36,1 \%$ e de $13,3 \%$, sem diferença estatística significativa em relação aos filhos de adultas. Crianças, amamentadas ou não, cujas mães tinham mais de 20 anos, consumiram outros leites em maior proporção que os filhos de mulheres mais jovens, com diferenças 
significativas. No primeiro semestre de vida, o uso de mamadeiras e chupetas foi semelhante entre filhos de adolescentes e adultas.

As práticas alimentares que ocorreram no primeiro dia em casa, após a alta hospitalar, estão apresentadas na Tabela 3. A oferta de leite materno foi proporcionalmente maior aos filhos de adolescentes em relação aos filhos de mulheres com mais de 20 anos, sem diferenças significativas. O uso de água e chás foi menor entre adolescentes, embora sem significância estatística. O consumo de outros leites, entretanto, foi maior entre filhos de adultas, com diferença estatística limítrofe $(p<0,10)$.
O perfil da amamentação, da oferta de outros leites e da alimentação complementar, entre maiores de seis meses, está apresentado na Tabela 4. Inversamente ao encontrado entre as crianças menores de 6 meses, a oferta de leite materno aos filhos de adolescentes foi significativamente menor, em relação aos filhos de mulheres adultas $(49,2 \%$ e $66,0 \%$, respectivamente; $p<0,05)$. Alimentos complementares foram menos consumidos por filhos de mulheres/ mães/adolescentes do que por filhos de adultas, embora as diferenças estatísticas apresentem significância limítrofe $(p<0,10)$. Mamadeiras foram mais usadas por mães adolescentes do que

Tabela 1. Características do conjunto de crianças e mães estudadas e prevalência de aleitamento materno no primeiro ano, segundo as faixas etárias maternas. Volta Redonda (RJ), 2006.

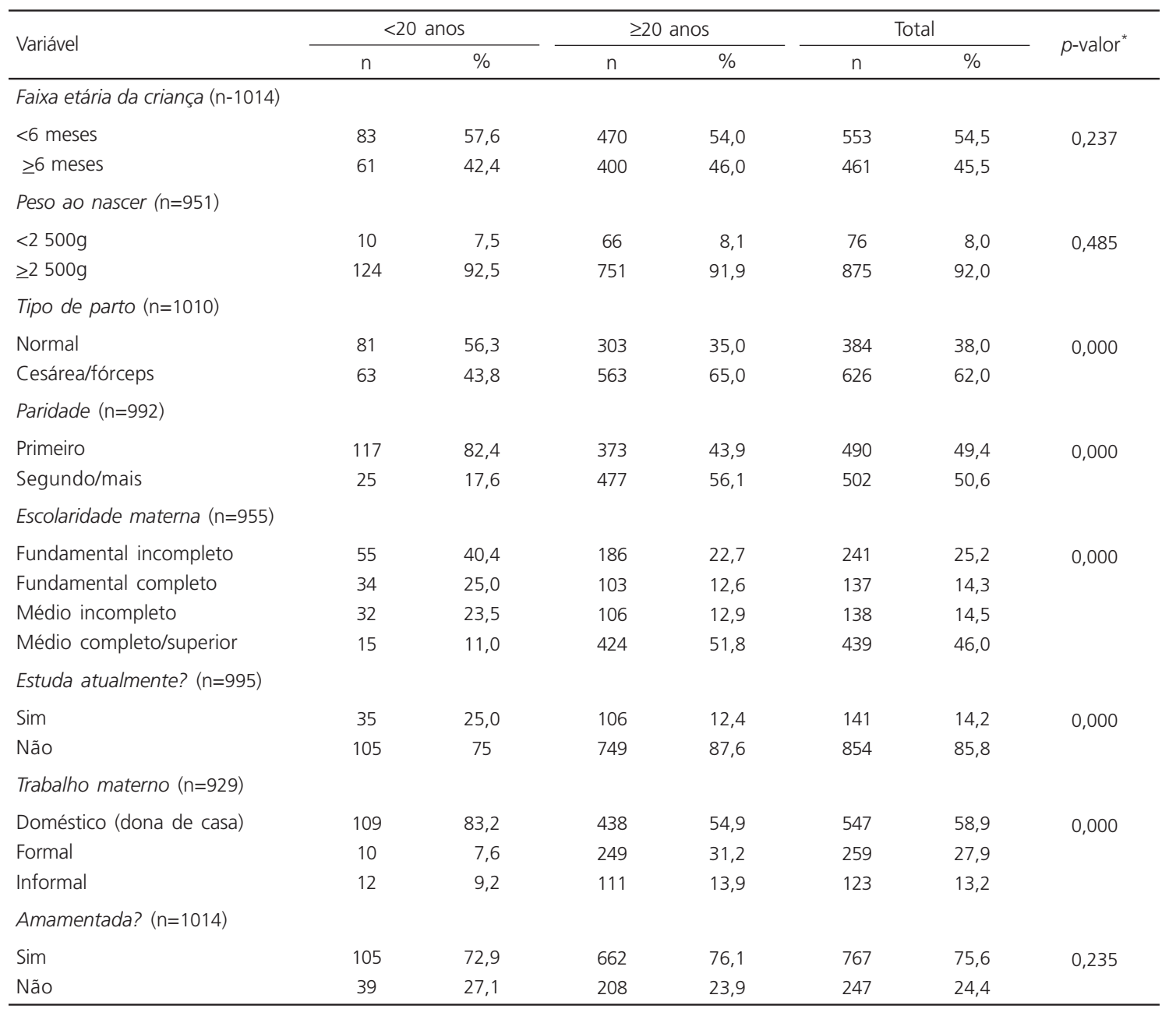

* Teste qui-quadrado. 
por adultas, diferença sem significância estatística. Observou-se um maior uso de chupetas por filhos de adolescentes, com diferenças significativas estatisticamente frente às adultas. Junto às crianças com idades de seis a nove meses, a alimentação complementar oportuna esteve presente entre $50,0 \%$ das crianças nos dois grupos (não exibido na tabela).

Quanto às características das refeições salgadas, observou-se o predomínio na oferta de

Tabela 2. Perfil de aleitamento materno das crianças menores de seis meses, segundo faixa etária materna. Volta Redonda (RJ), 2006.

\begin{tabular}{|c|c|c|c|c|c|c|c|}
\hline \multirow{2}{*}{ Variável } & \multicolumn{2}{|c|}{$<20$ anos } & \multicolumn{2}{|c|}{$\geq 20$ anos } & \multicolumn{2}{|c|}{ Total } & \multirow{2}{*}{$p$-valor* } \\
\hline & $\mathrm{n}$ & $\%$ & $\mathrm{n}$ & $\%$ & $n$ & $\%$ & \\
\hline \multicolumn{8}{|c|}{ Aleitamento materno $(n=553)$} \\
\hline Sim & 75 & 90,4 & 398 & 84,7 & 473 & 85,5 & 0,115 \\
\hline Não & 8 & 9,6 & 72 & 15,3 & 80 & 14,5 & \\
\hline \multicolumn{8}{|c|}{ Aleitamento materno exclusivo $(n=553)$} \\
\hline Sim & 30 & 36,1 & 147 & 31,3 & 177 & 32,0 & 0,226 \\
\hline Não & 53 & 63,9 & 323 & 68,7 & 376 & 68,0 & \\
\hline \multicolumn{8}{|c|}{ Aleitamento materno predominante $(n=553)$} \\
\hline Sim & 11 & 13,3 & 42 & 8,9 & 53 & 9,6 & 0,152 \\
\hline Não & 72 & 86,7 & 428 & 91,1 & 500 & 90,4 & \\
\hline \multicolumn{8}{|c|}{ Outros leites $(n=523)$} \\
\hline Sim & 24 & 31,2 & 202 & 45,3 & 226 & 43,2 & 0,014 \\
\hline Não & 53 & 68,8 & 244 & 54,7 & 297 & 56,8 & \\
\hline \multicolumn{8}{|c|}{ Mamadeira $(n=522)$} \\
\hline $\operatorname{Sim}$ & 37 & 47,4 & 197 & 44,4 & 234 & 44,8 & 0,352 \\
\hline Não & 41 & 52,6 & 247 & 55,6 & 288 & 55,2 & \\
\hline \multicolumn{8}{|c|}{ Chupeta $(n=541)$} \\
\hline Sim & 39 & 47,6 & 215 & 46,8 & 254 & 47,0 & 0,499 \\
\hline Não & 43 & 52,4 & 244 & 53,2 & 287 & 53,0 & \\
\hline
\end{tabular}

* Teste qui-quadrado.

Tabela 3. Tipo de alimentação fornecida no primeiro dia em casa, aos lactentes menores de 4 meses, filhos de adolescentes e adultas. Volta Redonda (RJ), 2006

\begin{tabular}{|c|c|c|c|c|c|c|c|}
\hline \multirow{2}{*}{ Variável } & \multicolumn{2}{|c|}{$<20$ anos } & \multicolumn{2}{|c|}{$\geq 20$ anos } & \multicolumn{2}{|c|}{ Total } & \multirow{2}{*}{$p$-valor ${ }^{*}$} \\
\hline & $\mathrm{n}$ & $\%$ & $\mathrm{n}$ & $\%$ & $n$ & $\%$ & \\
\hline \multicolumn{8}{|c|}{ Aleitamento materno $(n=362)$} \\
\hline Sim & 52 & 96,3 & 286 & 92,9 & 338 & 93,4 & 0,274 \\
\hline Não & 2 & 3,7 & 22 & 7,1 & 24 & 6,6 & \\
\hline \multicolumn{8}{|c|}{ Outro leite $(n=350)$} \\
\hline Sim & 7 & 13,0 & 67 & 22,6 & 74 & 21,1 & 0,073 \\
\hline Não & 47 & 87,0 & 229 & 77,4 & 276 & 78,9 & \\
\hline \multicolumn{8}{|c|}{ Água $(n=352)$} \\
\hline Sim & 2 & 3,7 & 20 & 6,7 & 22 & 6,2 & 0,314 \\
\hline Não & 53 & 96,3 & 278 & 93,3 & 330 & 93,8 & \\
\hline \multicolumn{8}{|c|}{ Chá $(n=349)$} \\
\hline Sim & 3 & 5,7 & 31 & 10,5 & 34 & 9,7 & 0,206 \\
\hline Não & 50 & 94,3 & 265 & 89,5 & 315 & 90,3 & \\
\hline
\end{tabular}

* Teste qui-quadrado. 
Tabela 4. Perfil da amamentação, da alimentação complementar e do uso de chupeta e mamadeira entre maiores de 6 meses, filhos de adolescentes e adultas. Volta Redonda (RJ), 2006

\begin{tabular}{|c|c|c|c|c|c|c|c|}
\hline \multirow{2}{*}{ Variável } & \multicolumn{2}{|c|}{$<20$ anos } & \multicolumn{2}{|c|}{$\geq 20$ anos } & \multicolumn{2}{|c|}{ Total } & \multirow{2}{*}{$p$-valor ${ }^{*}$} \\
\hline & $n$ & $\%$ & $n$ & $\%$ & $n$ & $\%$ & \\
\hline \multicolumn{8}{|c|}{ Aleitamento materno $(n=461)$} \\
\hline Não & 31 & 50,8 & 136 & 34,0 & 167 & 36,2 & \\
\hline \multicolumn{8}{|c|}{$A C(\mathrm{n}=461)$} \\
\hline Não & 33 & 54,1 & 172 & 43,0 & 205 & 44,5 & \\
\hline \multicolumn{8}{|c|}{ Mamadeira $(n=448)$} \\
\hline Sim & 42 & 70,0 & 244 & 63,4 & 286 & 64,3 & 0,198 \\
\hline Não & 18 & 30,0 & 141 & 36,6 & 159 & 35,7 & \\
\hline
\end{tabular}

* Teste qui-quadrado.

sopas ou papas $(79,5 \%)$. Preparações mais consistentes (comida) foram oferecidas a 53,2\% das crianças, sem diferenças estatisticamente significativas entre filhos de adolescentes e adultas. A forma mais frequente de servir as sopas ou papas foi amassada $(67,5 \%)$, seguida pela oferta em pedaços $(16,2 \%)$ e liquidificada $(11,7 \%)$. Carne e feijão, importantes fontes de proteínas e ferro, foram preferencialmente consumidos em refeições sólidas (comida), tanto por filhos de adolescentes, quanto por filhos de adultas.

\section{I S C U S S Ã O}

O estudo em questão evidenciou que as práticas de $\mathrm{AM}$ e $\mathrm{AME}$, no município de Volta Redonda, foram mais frequentes que as verificadas na região Sudeste e em todo o país. Entre crianças menores de seis meses, adolescentes e adultas amamentaram seus filhos de forma semelhante. No entanto, a proporção de crianças maiores de seis meses amamentadas foi menor entre filhos de adolescentes. $\mathrm{O}$ uso de chupetas mostrou-se mais frequente no conjunto de filhos de adolescentes no segundo semestre de vida.

A analise das prevalências do aleitamento materno exclusivo, em Volta Redonda, aos 30 e 120 dias (49,0\% e $27,4 \%$, respectivamente), revela que elas foram superiores às encontradas no Brasil (47,5\% e 17,5\%, respectivamente) e no Sudeste $(38,2 \% \text { e } 14,5 \% \text {, respectivamente) })^{13}$. Aos 120 dias, no entanto, foi inferior aos $61,2 \%$ verificados em Lins $(S P)^{17}$. A prevalência média do aleitamento materno exclusivo aos 6 meses (de $32,0 \%$ ) foi semelhante ao encontrado no estado de São Paulo. No entanto, entre menores de um ano, a prevalência de aleitamento de foi de $75,6 \%$, superior à encontrada por Venâncio et al. ${ }^{18}$, em São Paulo. Tal situação assemelha-se às observações de Sena et al. ${ }^{19}$, ao constatarem, no Distrito Federal, elevada prevalência do aleitamento materno, a despeito do curto período de amamentação exclusiva.

Nos primeiros seis meses, as semelhanças nas prevalências de aleitamento materno, aleitamento materno exclusivo ou predominante, nos grupos de mães adolescentes e adultas, corrobora com os resultados encontrados por Vieira ${ }^{20}$.

O aleitamento exclusivo no primeiro dia em casa é associado, positivamente, à continuidade do aleitamento exclusivo, ao passo que a oferta de outros leites, água e chás repercute negativamente no estabelecimento do aleitamento materno exclusivo ${ }^{21}$. No primeiro dia em casa, a oferta de leite materno foi maior aos filhos de adolescentes. Inversamente, a oferta de água, 
chás e outros leites foi superior ao verificado junto aos filhos de mulheres adultas, com diferenças não significativas. Vale destacar as observações de Audi et al. ${ }^{22}$, em Itapira, que verificaram, no primeiro dia em casa, que adolescentes primíparas tiveram maior chance de amamentar exclusivamente. Ressaltam-se também as observações de Lamounier et al. ${ }^{21}$, ao verificarem nas adolescentes, no pós-parto em Belo Horizonte (MG), que a decisão de não amamentar esteve relacionada à não alfabetização, à gestação não desejada, ao uso de drogas na gestação, à insatisfação quanto ao sexo do filho e ao pensamento em abortar durante a gestação estudada. A gemelaridade, a condição de alto risco do bebê e o medo de ele ficar retido no berçário foram outras variáveis, associadas ao filho, com impacto negativo sobre a amamentação. Frota \& Marcopito ${ }^{23}$, em Montes Claros (MG), verificaram, aos seis meses de vida da criança, que o desmame foi maior no conjunto de mães adolescentes com vida conjugal e com atividade fora do lar após o parto ${ }^{23}$.

As recomendações atuais sobre alimentação infantil destacam a continuidade da amamentação até os dois anos ou mais. No segundo semestre de vida, verifica-se, no entanto, a maior ocorrência de descontinuidade precoce do aleitamento materno entre adolescentes em relação às adultas, com diferenças significativas. Esses resultados reiteram os achados que apontam maiores riscos de desmame precoce dos filhos de adolescentes ${ }^{24}$.

A prevalência média de aleitamento materno no período de seis a doze meses foi de $63,8 \%$, enquanto que, no estado de São Paulo, foi de $50,0 \%{ }^{25}$.

Do primeiro para o segundo semestre de vida, observa-se uma redução de $45,6 \%$ nas prevalências de aleitamento materno de filhos de adolescentes. Com respeito aos filhos de maiores de 20 anos, a redução foi de $22,1 \%$. Essa situação foi acompanhada pelo aumento na frequência no uso de mamadeiras pelos maiores de seis meses, sem diferenças entre filhos de adolescentes e adultas.
Chama a atenção o uso de chupetas por filhos de adolescentes no segundo semestre de vida, cujo comportamento mostrou-se significativamente maior nesse grupo em relação às não adolescentes. A associação do uso de chupeta nos primeiros meses e a interrupção precoce do aleitamento está descrita na literatura. Como justificativa para esse fato, tem sido apontada a confusão de bicos ${ }^{26}$. O uso da chupeta, como um calmante para a criança e uma ajuda para a mãe, passados pelas gerações, e uma necessidade para os pais, foi identificado por Masson ${ }^{27}$, a despeito dos conhecimentos sobre as contra-indicações de seu uso. Deve-se questionar os motivos para o aumento no seu uso por filhos de adolescentes nessa fase.

$\mathrm{Katz}^{28}$ evidencia que as mães adolescentes, na experiência com amamentação, repetem mitos, crenças e valores presentes nas falas das mulheres em geral. Silva ${ }^{7}$ observa que a menor duração da amamentação das adolescentes pode ser explicada pela inexperiência, mas também pode indicar uma flutuação de valores sobre amamentação. As jovens mães podem estar captando primeiro e com mais rapidez as mudanças sociais frente às concepções sociais sobre aleitamento, em função de sua sensibilidade ${ }^{7}$. Durhand ${ }^{29}$ chama a atenção para o fato de que o reconhecimento como boa mãe consiste em uma demanda maior para as adolescentes, uma vez que são socialmente consideradas despreparadas e imaturas para a amamentação. Vale serem ressaltadas, no entanto, as observações de Horta et al. ${ }^{30}$ de que a duração da amamentação pelas mães adolescentes poderia estar relacionada à duração de sua amamentação quando crianças.

O período entre seis e nove meses marca a introdução de novos alimentos na dieta infantil. A prevalência de alimentação complementar oportuna foi semelhante à verificada no estado de São Paulo, de $48 \%{ }^{24}$. Quanto à consistência da alimentação complementar, ressalta-se, junto às crianças de 6 a 12 meses, o predomínio do consumo de sopas ou papas, preparações insuficientes energeticamente, realidade semelhante à encontrada por Saldiva et al. ${ }^{25}$, em São Paulo. Contrariamente, em Volta Redonda, a ingestão 
de comida de panela foi proporcionalmente inferior à encontrada pelos autores. Merece ser destacado que o consumo de alimentos de baixa densidade energética pode comprometer o crescimento e o desenvolvimento infantil. Isso se verifica em função do eficiente mecanismo de auto-regulação da ingestão diária de energia das crianças pequenas $^{10}$.

A oferta similar de alimentação complementar a filhos de adolescentes e não adolescentes corrobora Vieira et al..$^{20}$, inclusive quanto à maior disponibilidade de alimentos protéicos mais baratos aos filhos de adolescentes.

\section{O N C L U S Ã O}

As prevalências de aleitamento materno aos 30 e 120 dias, assim como ao longo do primeiro ano de vida, podem ser consideradas elevadas no município, quando comparadas às médias nacionais. Entretanto o aleitamento materno exclusivo nos primeiros 6 meses permanece próxima à observada no país. Essa realidade evidencia a complexidade da amamentação no cotidiano feminino. A formulação das ações de promoção, proteção e apoio à sua prática devem considerá-la como um híbrido que se constrói entre o domínio da biologia e da sociedade, respeitando as especificidades locais. Nessa direção, salienta-se a importância da escuta às mães, no intuito de conhecer tudo aquilo que elas percebem como obstáculo à sua prática.

Os resultados revelam o quanto as práticas alimentares de filhos de adolescentes e adultas, no primeiro ano de vida, distanciam-se das recomendações oficiais, tanto no que se refere ao aleitamento materno, como no tocante à alimentação complementar. O predomínio na oferta de sopas ou papas pode ser caracterizado como preocupante em função dos riscos de ingestão insuficiente de energia pela criança. A menor adição de carne e feijão a essas preparações agrava a realidade, comprometendo a ingestão, em especial, de ferro e zinco. A fim de proporcionar segurança nutricional a essas crianças, recomenda-se maior atenção aos saberes que fundamentam a oferta de alimentos comple- mentares, reconstruindo-os à luz dos conhecimentos atuais sobre o tema.

A variável idade materna, adolescente ou não, não possibilitou identificar diferenças na prática do aleitamento materno no primeiro semestre de vida, e sim após este período. A maternidade na adolescência não pode ser considerada, de forma generalizada, uma condição que se associe à interrupção precoce da amamentação, assim como ao desenvolvimento de práticas alimentares inadequadas. Os resultados, no entanto, sinalizam para a necessidade de ampliar a compreensão do processo decisório da mulher/adolescente quanto à alimentação de seu filho. Com esse olhar, será possível delinear estratégias que possibilitem responder às demandas que comprometem a alimentação saudável na infância.

\section{A GRADECIMENTOS}

Aos servidores da Secretaria Municipal de Saúde de Volta Redonda e aos acadêmicos dos cursos de Nutrição, Enfermagem e Medicina do Centro Universitário de Volta Redonda que participaram desta pesquisa.

\section{COLABORADORES}

M.C.C. CRUZ e J.A.G. ALMEIDA participaram na concepção e no desenho do estudo, nas análises e na interpretação dos resultados. E.M. ENGSTROM participou na seleção dos dados, na análises e na interpretação dos resultados.

\section{REFERÊ NCIAS}

1. Vieira GO, Silva LR, Vieira TO. Alimentação infantil e morbidade por diarréia. J Pediatr. 2006; 79(5): 449-54. doi: 10.1590/S0021-755720030005 00013

2. Horta BL, Bahl R, Martines JC, Victora CG. Evidence of long-term effects of breastfeeding: systematic reviews and meta-analyses. Geneva: WHO; 2007.

3. Augusto RA, Souza JMP. Crescimento de crianças em aleitamento materno exclusivo no primeiro semestre de vida. Rev Bras Crescimento Desenv Hum. 2007; 17(2):1-11. 
4. Vieira GO, Silva LR, Vieira TO, Almeida JAG, Cabral VA. Hábitos alimentares de crianças menores de 1 ano amamentadas e não amamentadas. J Pediatr. 2004; 80(5):411-6. doi: 10.1590/S0021-755720 04000600013.

5. Rotenberg S, De Vargas S. Práticas alimentares e o cuidado da saúde: da alimentação da criança à alimentação da família. Rev Bras Saúde Matern Infant. 2004; 4(1):85-94. doi: 10.1590/S1519-382 92004000100008

6. World Health Organization. Complementary feeding in the WHO Multicentre Growth Reference Study. Acta Pediatrica. 2006; (Suppl 450):27-37. doi: 10.1080/08035320500495456.

7. Silva AAM. Amamentação: fardo ou desejo? Estudo histórico-social dos saberes e práticas sobre aleitamento na sociedade brasileira [mestrado]. Ribeirão Preto: Universidade de São Paulo; 1990.

8. World Health Organization. Complementary feeding: report of the global consultation of guiding principles for complementary feeding of the breastfed child. Geneva: WHO; 2002.

9. Brasil. Ministério da Saúde. Guia alimentar para crianças menores de dois anos. Brasília: Ministério da Saúde; 2002. Série A. Normas e Manuais Técnicos.

10. Monte CMG, Giugliani E. Recomendações para a alimentação complementar em crianças em aleitamento materno. J Pediatr. 2004; 80(5 Supl): S131-41.

11. Batista Filho M, Rissin A. A transição nutricional: tendências regionais e temporais. Cad Saúde Pública. 2003; 19(1):S181-91. doi: 10.1590/S010 2-311X2003000700019.

12. Leite IC, Cruz MCC. Fatores de risco para déficits estaturais no segundo ano de vida: Brasil, PNDS, 1996. Rev Bras Est Pop. 2002: 19(1):131-40.

13. Sena MCF, Silva EF, Pereira MG. Prevalência do aleitamento materno nas capitais brasileiras. Rev Assoc Med Bras. 2007; 53(6):520-4. doi: 10.1590/S0 104-42302007000600020.

14. Brasil. Ministério da Saúde. Pesquisa nacional sobre demografia e saúde da criança e da mulher. Brasília; 2006 [acesso 2008 jul 9]. Disponível em: <http://bv sms.saude.gov.br/bvs/pnds/saude_nutricional.php >.

15. Instituto de Saúde de São Paulo. Projeto amamentação e municípios. São Paulo; 2006 [acesso 2008 jul 9]. Disponível em: <www.isaude.sp.gov.br/ama mu/rio/amario.htm>.

16. World Health Organization. Indicators for assessing infant and young child feeding practices: conclusions of a consensus meeting held 6-8 November 2007. Washington (DC): WHO; 2008.

17. Silva AP, Souza N. Prevalência de aleitamento materno. Rev Nutr. 2005; 18(3):301-10. doi: 10.1590/\$1 415-52732005000300002.
18. Venâncio SI, Escuder MML, Kitoco P, Rea MF, Monteiro CA. Frequência e determinantes do aleitamento materno em municípios do Estado de São Paulo. Rev Saúde Pública. 2002; 36(3):313-8. doi: 10.1590/S0034-89102002000300009.

19. Sena MCF, Silva EF, Pereira MG. Prevalência do aleitamento materno no Distrito Federal, Brasil. Cad Saúde Pública. 2002; 18(3):613-21. doi: 10.1590/S0102-311X2002000300010.

20. Vieira MLF, Pinto e Silva JLC, Barros Filho AA. A amamentação e a alimentação complementar de filhos de mães adolescentes são diferentes das de filhos de mães adultas? J Pediatr. 2003; 79(4):317-24. doi: 10.1590/S0021-75572003000400009.

21. Lamounier JA, Cardoso AMJ, Abrantes MM, Silva AA, Faria JF, Diniz CM. Fatores associados com o aleitamento materno em mães adolescentes. Rev Méd Minas Gerais. 2003; 13(4 Supl 2):S27-S30.

22. Audi CAF, Corrêa MAS, Latorre MRDO, PérezEscamilla R. Factors associated with infant feeding practices after hospital discharge. Rev Saúde Pública. 2005; 39(3):406-12. doi: 10.1590/S0034-89 102005000300011.

23. Frota DAL, Marcopito LF. Breastfeeding among teenage and adult mothers in Brazil. Rev Saúde Pública. 2004; 38(1):85-92. doi: 10.1590/S0034-89 102004000100012.

24. Chaves R, Lamounier JA, César CC. Factors associated with duration of breastfeeding. J Pediatr. 2007; 83(3):241-46.

25. Saldiva SRDM, Escuder MM, Mondini L, Levy RB, Venâncio SI. Feeding habits of children aged 6 to 12 months and associated maternal factors. J Pediatr. 2007; 83(1):53-8.

26. Contrim LC, Venâncio SI, Escuder MML. Uso de chupeta e amamentação em crianças menores de quatro meses no estado de São Paulo. Rev Bras Saúde Materno Infant. 2002; 2(3):245-52. doi: 10.1590/S1519-38292008000400003.

27. Masson SC. Representações sociais de mães sobre a chupeta [dissertação]. São Paulo: Universidade de São Paulo; 2002.

28. Katz RA. Adolescentes e maternidade: um destino, um problema, uma escolha? [dissertação]. Rio de Janeiro: Instituto Fernandes Figueira; 1999.

29. Durhand SB. Mães adolescentes: estudo das vivências de amamentação dos seus filhos [dissertação]. Rio de Janeiro: Universidade do Estado do Rio de Janeiro; 2007.

30. Horta BL, Victora CG, Gigante DP, Santos J, Barros FC. Duração da amamentação em duas gerações. Rev Saúde Pública. 2007; 41(1):13-18. doi: 10.15 90/S1519-38292008000400003.

Recebido em: 30/7/2008

Versão final reapresentada em: 15/4/2009 Aprovado em: 6/10/2009 